\title{
Stability Analysis of Flow in Thin Liquid Film Under Heat Effect \\ Zena Talal Al-Nuamy
}

zana-talal@uomosul.edu.iq

College of computer sciences and Mathematics

University of Mosul, Mosul, Iraq

\section{Received on: 27/10/2010}

ABSTRACT

Accepted on: 16/3/2011

This research is dedicated for analyzing the stability of system of flow equations for a delicate chip of a fluid exposed for medium heat, where we found the growth of disturbance exist or not after making the system linearization it was shown through the results of analysis that these equations were in stable state when the real part of the wave velocity $(\alpha)$ is a negative quantity and it is unstable state when the real part of this velocity is positive quantity, We get stability and this happens when the Reynold's number is positive quantity i.e. $\mathrm{R}>0$. and when $\alpha>0$ we get unstable state and this happens when the heat conductivity is a positive quantity.

Keywords: Stability Analysis, Dimensional analysis, System Linearization, Wave velocity, Reynold's number

$$
\begin{aligned}
& \text { تحليل الاستقرارية لجريان شريحة رقيقة لمائع تحت تأثير الحرارة } \\
& \text { زينة طلال النعيمي } \\
& \text { كلية علوم الحاسوب والرياضيات، جامعة الدوصل طبل }
\end{aligned}
$$

تاريخ قبول البحث: 16 / 3 / 2011

تاريخ استلام البحث: 27 / 10 / 2010

\section{الملخص}

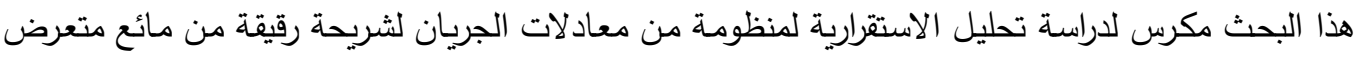

لتأثير حرارة الوسط إذ تمكنا من إيجاد نمو الاضطراب من عدمه وذلك بعد جعل النظام خطي. وتبين من نتائج التحليل

أن هذه المعادلات تكون مستقرة عندما يكون الجزء الحقيقي لسرعة الموجة (م) كمية سالبة، وتكون غير مستقرة عندما

يكون الجزء الحقيقي لهذه السرعة كمية موجبة نحصل على الاستقرار وهذا يحدث عندما يكون عدد رينولد كمية موجبة

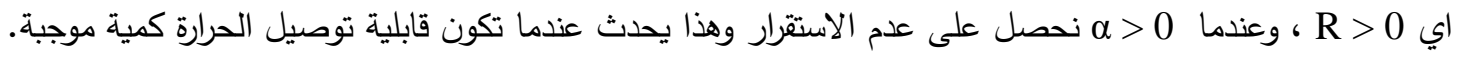

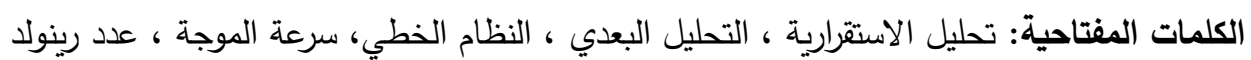

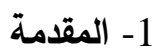

توفر الدراسات التي أجريت على استقرارية الثرائح الرقيقة للموائع معلومات قيمة عن الاستخدامات المتعددة

$$
\text { في الهندسة الكيميائية والميكانيكية والبيولوجية. }
$$

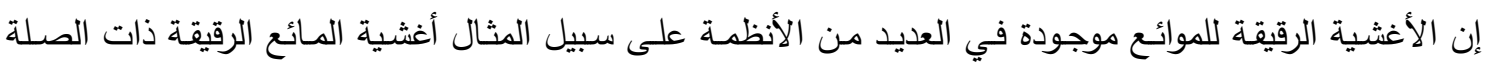
بسلوكية الطفو ورغوة واندماج الفقاعات وقد تكون الشريحة الرقيقة من المائع غير مستقرة عندما تصبح رقئه التيقة جداً.

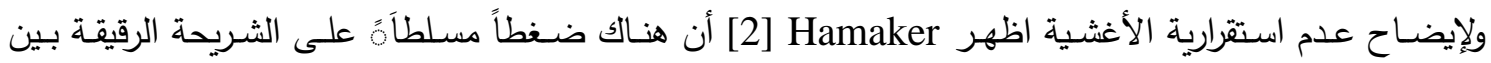
طورين مكثين لذلك فان الطاقة لكل وحدة حجم تختلف عن الطاقة التي تتولد من الطور الحجمي للمادة التي تؤلف شريحة المائع. - مان.

أما Scheludko [2] فقد درس عدم استقرارية الثربحة الرقيقة بسبب تضخيم الذبذبات التلقائية بواسطة

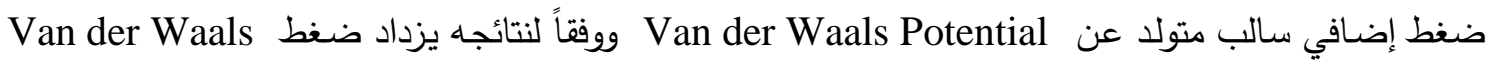
غير الرابط مع تتاقض سمك الثريحة وإذا ما تم تثويش الثريحة الرقيقة فان الضغط غير الرابط السالب يحدث

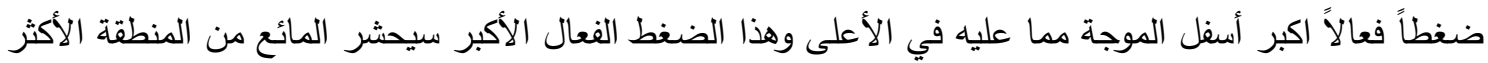


رقة إلى المنطقة الأكثر سمكاً للشريحة الرقيقة وعند حدوث هذا الحشر ستقل سماكة الثريحة بصورة مستمرة حتى

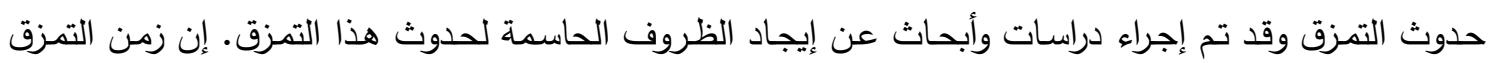

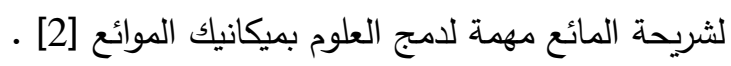
وفي العام 1916 م قام Nusselt [11] بدراسة تكثيف البخار على سطح عمودي ومنذ ذلك الحين أجريت دراسات عديدة عن تكثيف وتبخير الثرائح الرقيقة للموائع وتحت تأثير ظروف مختلفة حيث أن غالبية الدراسات

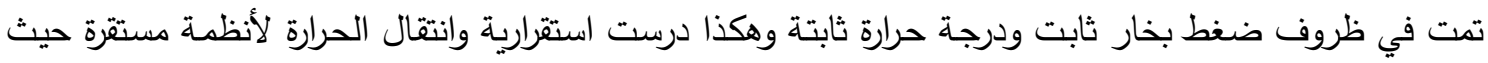
تعد الشريحة باتجاه الأسفل غير مستقرة بسبب عدم استقرارية Reyleigh - Taylor وعدم الاستقرارية هذه تتتج

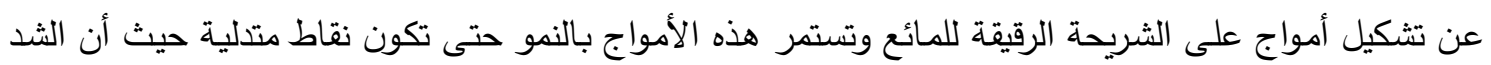

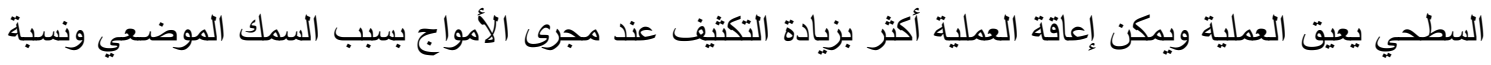

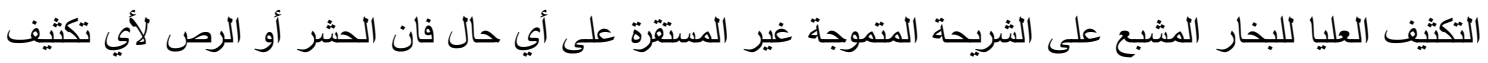

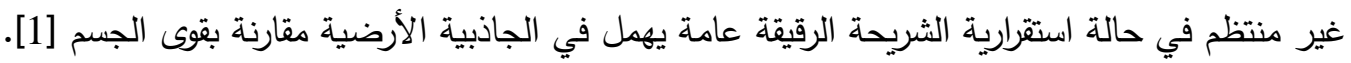

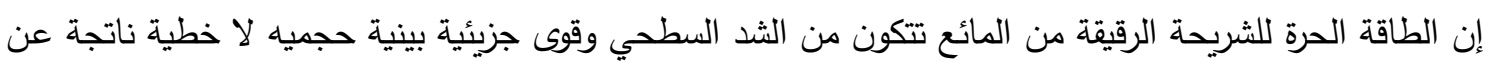

Van der Waals اظهر تحليل الاستقرارية الثيرموديناميكية أن الثد السطحي يزيد من استقرارية الشريحة بينما قوى Van der

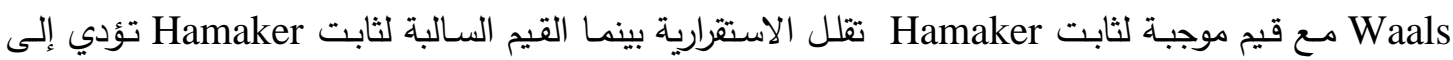

[8] الاستقرارية

إن مجالات تطبيق هذا البحث تعتمد على أنظمة تكثيف الأبخرة حيث تستخدم هذه الأنظمة في مجالات

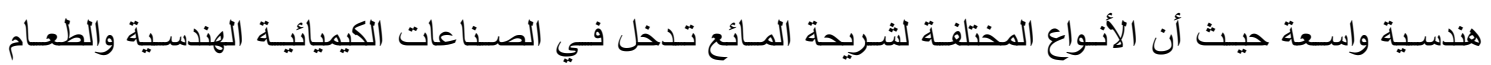
والصناعات الدوائية، حيث انه من الضروري الحفاظ على مصادر الطاقة وذلك لتحسين كمية الإنتاج ولزيادة كفاءة أنظمـة التبخير والتكثيف ويمكن التغلب على هذه المشاكل بسهولة عن طريق نمذجـة التبادل الحراري للمائع في

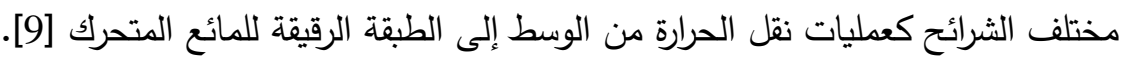

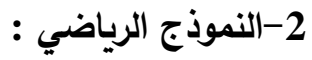
ليكن لاينا مائع على شكل شريحة رقيقة غير قابلة للانضغاط وغير معرضـة إلى قوى خارجية وتحت تأثير حرارة الوسط في النظام ثنائي البعد. وبفرض أن الشريحة متتاظرة مع الإحداثي العمودي سنحصل على المعادلات

$$
\begin{aligned}
& \frac{\partial u^{\prime}}{\partial t^{\prime}}+u^{\prime} \frac{\partial u^{\prime}}{\partial x^{\prime}}+v^{\prime} \frac{\partial u^{\prime}}{\partial y^{\prime}}=-\frac{1}{\rho} \frac{\partial p^{\prime}}{\partial x^{\prime}}+\frac{\mu}{\rho}\left(\frac{\partial^{2} u^{\prime}}{\partial x^{\prime 2}}+\frac{\partial^{2} u^{\prime}}{\partial y^{\prime 2}}\right) . \\
& \frac{\partial v^{\prime}}{\partial t^{\prime}}+u^{\prime} \frac{\partial v^{\prime}}{\partial x^{\prime}}+v^{\prime} \frac{\partial v^{\prime}}{\partial y^{\prime}}=-\frac{1}{\rho} \frac{\partial p^{\prime}}{\partial y^{\prime}}+\frac{\mu}{\rho}\left(\frac{\partial^{2} v^{\prime}}{\partial x^{\prime^{2}}}+\frac{\partial^{2} v^{\prime}}{\partial y^{\prime 2}}\right) .
\end{aligned}
$$
إن صيغة معادلة الاستمرارية للجريان غير قابل للانضغاط في النظام الثنائي البعد هي:

$$
\frac{\partial u^{\prime}}{\partial x^{\prime}}+\frac{\partial v^{\prime}}{\partial y^{\prime}}=0
$$

$$
\frac{\partial T}{\partial t}+u^{\prime} \frac{\partial T}{\partial x^{\prime}}+v^{\prime} \frac{\partial T^{\prime}}{\partial y^{\prime}}=a\left[\frac{\partial^{2} T^{\prime}}{\partial x^{\prime^{2}}}+\frac{\partial^{2} T^{\prime}}{\partial y^{\prime 2}}\right]
$$




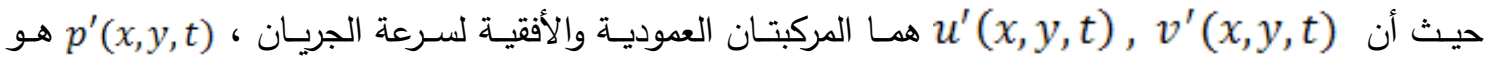
الضغطو لم تمثل لزوجة المائع و $\rho$ هي الكثافة ،

في نظريـة التزييـت يكـون الحـد فرارة الحرارة $\frac{\partial u^{\prime}}{\partial t^{\prime}}+u^{\prime} \frac{\partial u^{\prime}}{\partial x^{\prime}}+v^{\prime} \frac{\partial u^{\prime}}{\partial y^{\prime}}=-\frac{1}{\rho} \frac{\partial p^{\prime}}{\partial x^{\prime}}+\frac{\mu}{\rho}\left(\frac{\partial^{2} u^{\prime}}{\partial y^{\prime 2}}\right)$

$\frac{\partial v^{\prime}}{\partial t^{\prime}}+u^{\prime} \frac{\partial v^{\prime}}{\partial x^{\prime}}+v^{\prime} \frac{\partial v^{\prime}}{\partial y^{\prime}}=-\frac{1}{\rho} \frac{\partial p^{\prime}}{\partial y^{\prime}}+\frac{\mu}{\rho}\left(\frac{\partial^{2} v^{\prime}}{\partial y^{\prime 2}}\right)$

$\frac{\partial u^{\prime}}{\partial x^{\prime}}+\frac{\partial v^{\prime}}{\partial y^{\prime}}=0$

$\frac{\partial T^{\prime}}{\partial t^{\prime}}+u^{\prime} \frac{\partial T^{\prime}}{\partial x^{\prime}}+v^{\prime} \frac{\partial T^{\prime}}{\partial y^{\prime}}=a\left(\frac{\partial^{2} T^{\prime}}{\partial y^{\prime 2}}\right) \ldots \ldots \ldots$

3- التحليل البُعدي (Dimensional analysis):-

لغرض إيجاد المعادلات اللا بعدية للمعادلات (5)و و(6)و (7) و(8) سوف نفرض بعض القيم اللا بعدية

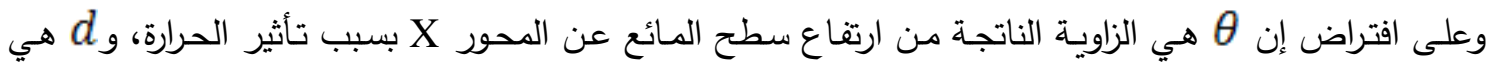
مسافة ثابتة معلومة وكالاتي [6] :

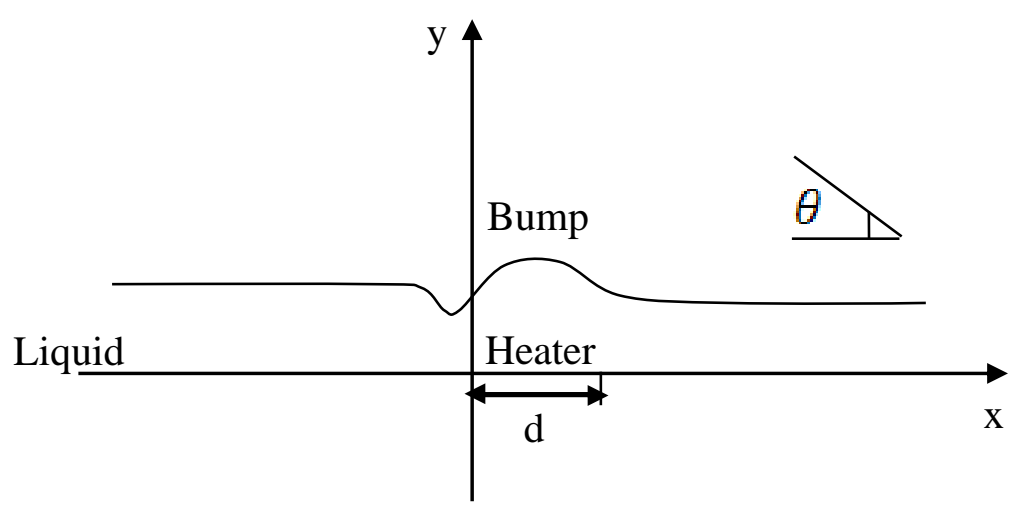

$x^{I}=x d, \quad y^{I}=y d, \quad t^{r}=t \sqrt{d / g}, \quad u^{r}=u \sqrt{d g}$

$v^{r}=v \sqrt{d g}, \quad p^{I}=p d g \quad, \quad T^{r}=\theta T$

وبتعويض الكميات والقيم اللا بعدية في المعادلات من (5) - (8) نحصل على المعادلات اللا بعدية لمعادلة طبقة

$$
\begin{aligned}
& \frac{\partial u}{\partial t}+u \frac{\partial u}{\partial x}+v \frac{\partial u}{\partial y}=-\frac{1}{\rho} \frac{\partial p}{\partial x}+\frac{\partial^{2} u}{\partial y^{2}} / R \\
& \frac{\partial v}{\partial t}+u \frac{\partial v}{\partial x}+v \frac{\partial v}{\partial y}=-\frac{1}{\rho} \frac{\partial p}{\partial y}+\frac{\partial^{2} v}{\partial y^{2}} / R \ldots
\end{aligned}
$$
رقيقة من مائع جاري تحت تأثير الحرارة وكالاتي: 
$\frac{\partial u}{\partial x}+\frac{\partial v}{\partial y}=0$

$\frac{\partial \theta}{\partial t}+u \frac{\partial \theta}{\partial x}+v \frac{\partial \theta}{\partial y}=K \frac{\partial^{2} \theta}{\partial y^{2}}$

$R=\frac{g \rho d^{2}}{\mu \sqrt{d g}}$

حيث أن Reynold's Number Rو Re ومعرف كالأتي [10]:

$K=\frac{a \sqrt{d / g}}{d^{2}}$

وان K هو قابلية توصيل الحرارة ومعرف كالأتي [9] :

4 - تحليل الاستقرارية (Stability Analysis)

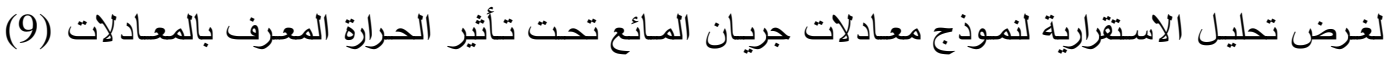

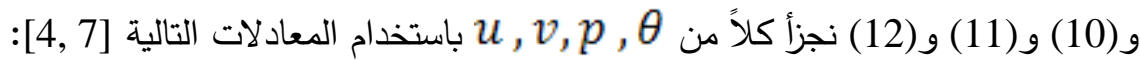

$u(x, y, t)=u_{1}(x)+u_{2}(x, y, t)$

$v(x, y, t)=v_{1}(x)+v_{2}(x, y, t)$

$p(x, y, t)=p_{1}(x)+p_{2}(\mathrm{x}, \mathrm{y}, \mathrm{t})$

$\theta(x, y, t)=\theta_{1}(x)+\theta_{2}(\mathrm{x}, \mathrm{y}, \mathrm{t})$

إذ أن

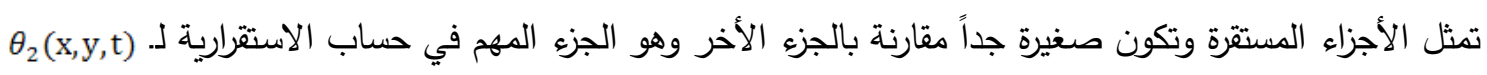

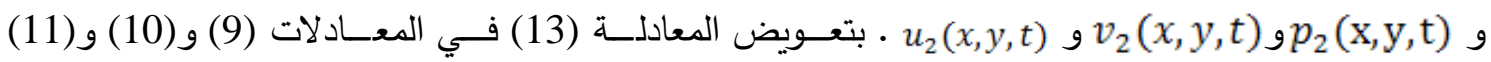

$\begin{aligned} \frac{\partial\left(u_{1}+u_{2}\right)}{\partial t} & +\left(u_{1}+u_{2}\right) \frac{\partial\left(u_{1}+u_{2}\right)}{\partial x}+\left(v_{1}+v_{2}\right) \frac{\partial\left(u_{1}+u_{2}\right)}{\partial y} \\ & =-\frac{1}{\rho} \frac{\partial\left(p_{1}+p_{2}\right)}{\partial x}+\left(\frac{\partial^{2}\left(u_{1}+u_{2}\right)}{\partial y^{2}}\right) / R \ldots \ldots \ldots .\end{aligned}$

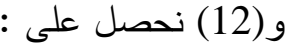

$$
\begin{aligned}
\frac{\partial\left(v_{1}+v_{2}\right)}{\partial t} & +\left(u_{1}+u_{2}\right) \frac{\partial\left(v_{1}+v_{2}\right)}{\partial x}+\left(v_{1}+v_{2}\right) \frac{\partial\left(v_{1}+v_{2}\right)}{\partial y} \\
& =-\frac{1}{\rho} \frac{\partial\left(p_{1}+p_{2}\right)}{\partial y}+\left(\frac{\partial^{2}\left(v_{1}+v_{2}\right)}{\partial y^{2}}\right) / R \ldots \ldots \ldots . .
\end{aligned}
$$

$\frac{\partial\left(u_{1}+u_{2}\right)}{\partial x}+\frac{\partial\left(v_{1}+v_{2}\right)}{\partial y}=0$

$$
\begin{aligned}
\frac{\partial\left(\theta_{1}+\theta_{2}\right)}{\partial t} & +\left(u_{1}+u_{2}\right) \frac{\partial\left(\theta_{1}+\theta_{2}\right)}{\partial x}+\left(v_{1}+v_{2}\right) \frac{\partial\left(\theta_{1}+\theta_{2}\right)}{\partial y} \\
& =K \frac{\partial^{2}\left(\theta_{1}+\theta_{2}\right)}{\partial y^{2}} \ldots \ldots \ldots(17)
\end{aligned}
$$


المعادلات (14) و(15) و(16) و(17) يمكن كتابتها على التوالي بالثكل:

$\frac{\partial u_{1}}{\partial t}+\frac{\partial u_{2}}{\partial t}+u_{1} \frac{\partial u_{1}}{\partial x}+u_{1} \frac{\partial u_{2}}{\partial x}+u_{2} \frac{\partial u_{1}}{\partial x}+u_{2} \frac{\partial u_{2}}{\partial x}+v_{1} \frac{\partial u_{1}}{\partial y}+v_{1} \frac{\partial u_{2}}{\partial y}$

$+v_{2} \frac{\partial u_{1}}{\partial y}+v_{2} \frac{\partial u_{2}}{\partial y}=-\frac{1}{\rho} \frac{\partial p_{1}}{\partial x}-\frac{1}{\rho} \frac{\partial p_{2}}{\partial x}+\frac{\partial^{2} u_{1}}{\partial y^{2}} / R+\frac{\partial^{2} u_{2}}{\partial y^{2}} / R \ldots$ (18)

$\frac{\partial v_{1}}{\partial t}+\frac{\partial v_{2}}{\partial t}+u_{1} \frac{\partial v_{1}}{\partial x}+u_{1} \frac{\partial v_{2}}{\partial x}+u_{2} \frac{\partial v_{1}}{\partial x}+u_{2} \frac{\partial v_{2}}{\partial x}+v_{1} \frac{\partial v_{1}}{\partial y}+v_{1} \frac{\partial v_{2}}{\partial y}$

$+v_{2} \frac{\partial v_{1}}{\partial y}+v_{2} \frac{\partial v_{2}}{\partial y}=-\frac{1}{\rho} \frac{\partial p_{1}}{\partial y}-\frac{1}{\rho} \frac{\partial p_{2}}{\partial y}+\frac{\partial^{2} v_{1}}{\partial y^{2}} / R+\frac{\partial^{2} v_{2}}{\partial y^{2}} / R \ldots$

$\frac{\partial u_{1}}{\partial x}+\frac{\partial u_{2}}{\partial x}+\frac{\partial v_{1}}{\partial y}+\frac{\partial v_{2}}{\partial y}=0$

$\frac{\partial \theta_{1}}{\partial t}+\frac{\partial \theta_{2}}{\partial t}+u_{1} \frac{\partial \theta_{1}}{\partial x}+u_{1} \frac{\partial \theta_{2}}{\partial x}+u_{2} \frac{\partial \theta_{1}}{\partial x}+u_{2} \frac{\partial \theta_{2}}{\partial x}+v_{1} \frac{\partial \theta_{1}}{\partial y}+v_{1} \frac{\partial \theta_{2}}{\partial y}$

$+v_{2} \frac{\partial \theta_{1}}{\partial y}+v_{2} \frac{\partial \theta_{2}}{\partial y}=K \frac{\partial^{2} \theta_{1}}{\partial y^{2}}+K \frac{\partial^{2} \theta_{2}}{\partial y^{2}}$

من المعلوم انه أي حد يحوي على حاصل ضرب الاستقرارية بالاضطراب سوف يكون غير مستقر لذلك

فان الحالة المستقرة في كل الكميات الموجودة في المعادلات يجب أن تكون بدلالة

$u_{1} \frac{\partial u_{1}}{\partial x}+u_{2} \frac{\partial u_{1}}{\partial x}=-\frac{1}{\rho} \frac{\partial p_{1}}{\partial x}$ أن المعادلات المستقرة ستتمثل بـ :

$u_{1} \frac{\partial v_{1}}{\partial x}+u_{2} \frac{\partial v_{1}}{\partial x}=0$

$\frac{\partial u_{1}}{\partial x}=0$

$u_{1} \frac{\partial \theta_{1}}{\partial x}+u_{2} \frac{\partial \theta_{1}}{\partial x}=0$

وعليه حتى نصف الجريان الأساسي نسبة إلى الاضطراب يجب أن نتحقق من إهمال الحدود الغير خطية

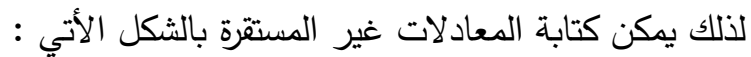

$\frac{\partial u_{2}}{\partial t}+u_{1} \frac{\partial u_{2}}{\partial x}=-\frac{1}{\rho} \frac{\partial p_{2}}{\partial x}+\left(\frac{\partial^{2} u_{2}}{\partial y^{2}}\right) / R$

$\frac{\partial v_{2}}{\partial t}+u_{1} \frac{\partial v_{2}}{\partial x}=-\frac{1}{\rho} \frac{\partial p_{2}}{\partial y}+\left(\frac{\partial^{2} v_{2}}{\partial y^{2}}\right) / R$

$\frac{\partial u_{2}}{\partial x}+\frac{\partial v_{2}}{\partial y}=0$

$\frac{\partial \theta_{2}}{\partial t}+u_{1} \frac{\partial \theta_{2}}{\partial x}=K \frac{\partial^{2} \theta_{2}}{\partial y^{2}}$

$v_{2}=0$ at $y=0, u_{2}=0$ at $y=0, \theta_{2}=0$ at $y=0$ 


\section{5- الاضطراب الحادث بالاتجاهين X,}

لإيجـاد الحل لهذه المنظومـة من المعـادلات نفرض إن الاضطراب حاصل بالاتجاهين X,Y وان السعة

ثابتة، يمكن كتابة المعادلات بالصورة التالية [7] :

$\left.\begin{array}{l}u_{2}=A_{1} e^{\alpha t+i\left(K_{1} x+K_{2} y\right)} \\ v_{2}=A_{2} e^{\alpha t+i\left(K_{1} x+K_{2} y\right)} \\ p_{2}=A_{3} e^{\alpha t+i\left(K_{1} x+K_{2} y\right)} \\ \theta_{2}=A_{4} e^{\alpha t+i\left(K_{1} x+K_{2} y\right)}\end{array}\right\} \ldots \ldots$

بحيث أن

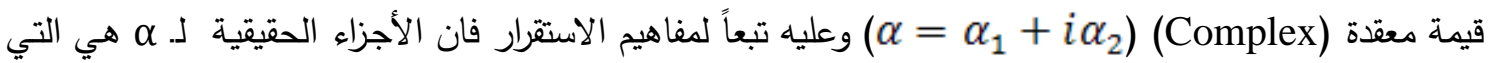
تؤدي إلى نمو الاضطراب أو تلاشيه على التوالي.

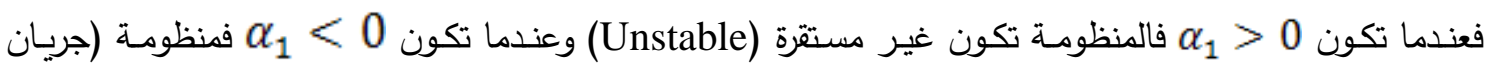

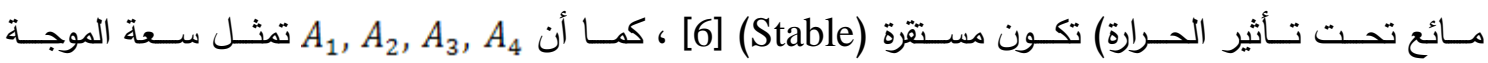

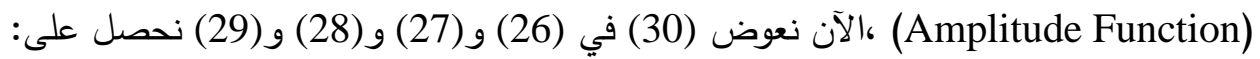
$A_{1}\left(i K_{1}\right) e^{\alpha t+i\left(K_{1} x+K_{2} y\right)}+A_{2}\left(i K_{2}\right) e^{\alpha t+i\left(K_{1} x+K_{2} y\right)}=0$

$\alpha A_{1} e^{\alpha t+i\left(K_{1} x+K_{2} y\right)}+u_{1} A_{1}\left(i K_{1}\right) e^{\alpha t+i\left(K_{1} x+K_{2} y\right)}=-\frac{1}{\rho} A_{3}\left(i K_{1}\right)$

* $e^{\alpha t+i\left(K_{1} x+K_{2} y\right)}+A_{1}\left(-K_{2}^{2}\right) / R * e^{\alpha t+i\left(K_{1} x+K_{2} y\right)}$

$\alpha A_{2} e^{\alpha t+i\left(K_{1} x+K_{2} y\right)}+u_{1} A_{2}\left(i K_{1}\right) e^{\alpha t+i\left(K_{1} x+K_{2} y\right)}=-\frac{1}{\rho} A_{3}\left(i K_{2}\right)$

$* e^{\alpha t+i\left(K_{1} x+K_{2} y\right)}+A_{2}\left(-K_{2}^{2}\right) / R * e^{\alpha t+i\left(K_{1} x+K_{2} y\right)}$

$\alpha A_{4} e^{\alpha t+i\left(K_{1} x+K_{2} y\right)}+u_{1} A_{4}\left(i K_{1}\right) e^{\alpha t+i\left(K_{1} x+K_{2} y\right)}=K A_{4}\left(-K_{2}^{2}\right) e^{\alpha t+i\left(K_{1} x+K_{2} y\right)}$

$e^{\alpha t+i\left(K_{1} x+K_{2} y\right)} \neq 0$

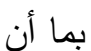

$i K_{1} A_{1}+i K_{2} A_{2}=0$

$\left(\alpha+i K_{1} u_{1}+K_{2}^{2} / R\right) A_{1}+\frac{1}{\rho}\left(i K_{1}\right) A_{3}=0$

$\left(\alpha+i K_{1} u_{1}+K_{2}^{2} / R\right) A_{2}+\frac{1}{\rho}\left(i K_{2}\right) A_{3}=0$

$\left(\alpha+i K_{1} u_{1}+K K_{2}^{2}\right) A_{4}=0$

حيث يمكن تمثيل المعادلات أعلاه بالمصفوفة التالية: 


$$
X=\left[\begin{array}{cccc}
\alpha+i K_{1} u_{1}+K_{2}^{2} / R & 0 & 1 / \rho * i K_{1} & 0 \\
0 & \alpha+i K_{1} u_{1}+K_{2}^{2} / R & 1 / \rho * i K_{2} & 0 \\
i K_{1} & i K_{2} & 0 & 0 \\
0 & 0 & 0 & \alpha+i K_{1} u_{1}+K K_{2}^{2}
\end{array}\right]
$$

وباستخدام برنامج خاص في نظام الـ (MATLAB) يمكن حساب [1]

$|X|(1$ 2) حل المعادلة 0 (

$$
\begin{aligned}
& |X|=\left(\alpha R+i K_{1} u_{1} R+K_{2}^{2}\right)\left(\alpha+i K_{1} u_{1}+K K_{2}^{2}\right)\left(K_{1}^{2}+K_{2}^{2}\right) / R / \rho \\
& |X|=0 \Longrightarrow
\end{aligned}
$$

$\left(\alpha R+i K_{1} u_{1} R+K_{2}^{2}\right)\left(\alpha+i K_{1} u_{1}+K K_{2}^{2}\right)\left(K_{1}^{2}+K_{2}^{2}\right) / R / \rho=0$

$\Longrightarrow$

$\alpha=-K_{2}^{2} K-i K_{1} u_{1}$

$\Longrightarrow$

$\alpha_{1}=-K_{2}^{2} K$

$\Rightarrow \alpha_{1}=-K_{2}^{2} / \mathrm{R}$
إما

: 2 نحصل على الاستقرارية في الحالات الآتية:

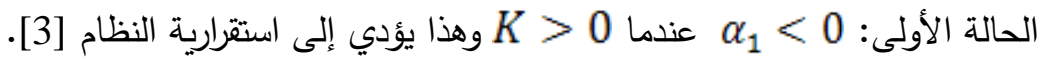

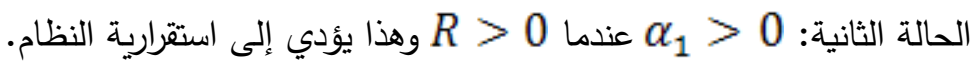
أما عدم الاستقرارية فنحصل عليها عندما تكون $R<0$ (1 $K<0$ (2

6- الاستنتاجات:-

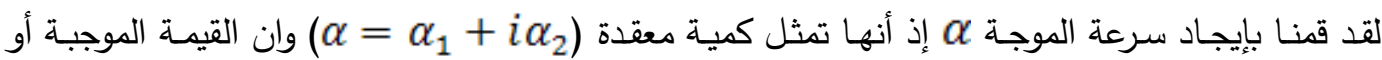
السالبة لـ $\alpha_{1}$ هي التي تؤثر في حالة الاضطراب أو تلاشيه. لقد تم تجزئة نظام المعادلات إلى جزئيين، الجزء الأول هو الجزء المستقر ويكون صغير جداً مقارنة بالجزء الثاني والمهم في تحليل الاستقرارية وتم إيجاد الحل أو فولئه قيم $\alpha$ باستخدام نظام الـ (MATLAB) والتي تعتمد على قيم Reynold's Number) و و $R$ و قابلية توصيل

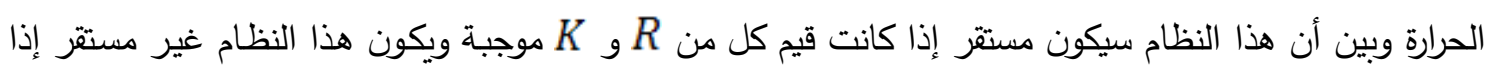
كانت قيم كل من R و $R$ ونالبة. 


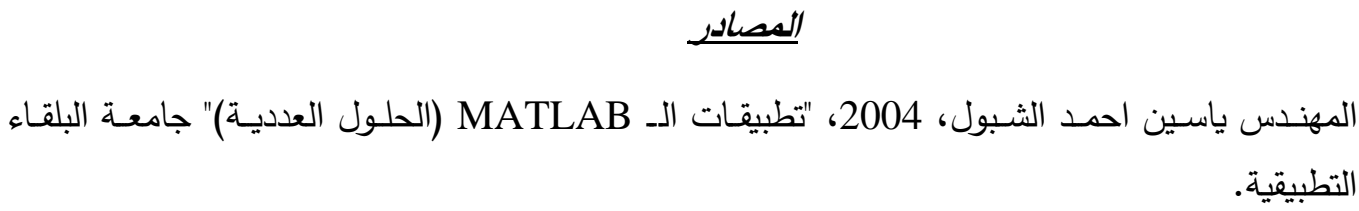

[2] Chi- Chuan Hwang and Jun-Liang Chen, 2005, "Stability of thin liquid films".

[3] Henry M.P. and Robert J.S., 1978, "Introduction to dynamics and control ", McGrew Hill, London.

[4] Ida M., 1996, "Dynamics and Stability of thin liquid films", proceeding of the $13^{\text {th }}$ Dundee conference of ordinary and partial differential equations, June 2528, Dundee, Scottand

[5] John M.Burgess, swift G.B., 1998, "Instability of the KoLmogorov flow in a soap film".

[6] Johnson M.F., Schluter R.A. and Bankoff S.G., 1996, "Stability and contact line motion of thin liquid films", national heat transfer conference.

[7] Logan J.D. 1987, "Applied mathematics", Wiley and Sony.

[8] Majumdar A. and Mezic I., 1998, "Stability Regimes of thin liquid films", Microscale thermophsical engineering, Vol. 2, pp. 203-213.

[9] Marchuk I. and Kabov O.A., 1998, "Numerical modeling of thermo capillary reverse flow in thin liquid films under local heating", Russ. J. Eng. Thermo physics,Vol. 8, No.1-4, P.17-46.

[10] Norman C., 2005, "Gas Bubble with a Moving Contact line rising in an Inclined channel at finite Reynold's Number", Physica P 209, Vol.8, No.1-4, PP. 17-46

[11] Sanjoy M. Som, Jeram T. Kimball, James C. Hermanson, Jeffrey S. Allen, 2007, "stability and heat transfer characteristics of unsteady condensing and evaporating films", S.M. Som et al. / International journal of heat and mass transfer Vol. 50, PP. 1927-1937. 